\title{
A New Similarity Measure of Generalized Trapezoidal Fuzzy Numbers and Its Application on Rotor Fault Diagnosis
}

\author{
Xin Zuo, Lijun Wang, and Yuanlong Yue \\ Department of Automation, China University of Petroleum-Beijing (CUPB), Beijing 102249, China \\ Correspondence should be addressed to Lijun Wang; wanglijun861130@163.com
}

Received 8 January 2013; Accepted 24 January 2013

Academic Editor: Valentina E. Balas

Copyright ( 2013 Xin Zuo et al. This is an open access article distributed under the Creative Commons Attribution License, which permits unrestricted use, distribution, and reproduction in any medium, provided the original work is properly cited.

\begin{abstract}
Fault diagnosis technology plays a vital role in the variety of critical engineering applications. Fuzzy approach is widely employed to cope with decision-making problems because it is in the simplest and most used form. This paper proposed a new similarity measure of generalized trapezoidal fuzzy numbers used for fault diagnosis. The presented similarity measure combines concepts of the geometric distance, the center of gravity point, the perimeter, and the area of the generalized trapezoidal fuzzy numbers for calculating the degree of similarity between generalized trapezoidal fuzzy numbers. This method is proposed to deal with both standardized and nonstandardized generalized trapezoidal fuzzy numbers. Some properties of the proposed similarity measure have been proved, and 12 sets of generalized fuzzy numbers have been used to compare the calculation results of the proposed similarity measures with the existing similarity measures. Comparison results indicate that the proposed similarity measure can overcome the drawbacks of existing similarity measures. Finally, a fault diagnosis experiment is carried out in laboratory based on multifunctional flexible rotor experiment bench. Experimental results demonstrate that the proposed similarity measure is more effective than other methods in terms of rotor fault diagnosis.
\end{abstract}

\section{Introduction}

Fault diagnosis technology has gained attention from researchers for its various applications [1-3]. To date, a large number of valuable approaches have been proposed for dealing with fault analysis issues, such as fuzzy theories $[4,5]$, expert system [6], wavelet analysis [7, 8], data fusion $[9,10]$, and neural network [11, 12]. Particularly, fuzzy approach is most successfully applied in fault diagnosis because it is in the simplest and most used form [13]. Using the fuzzy theories during the decision-making process, it is necessary to develop suitable technology to measure fuzzy similarity. Ranking the fuzzy numbers plays an important role in decisionmaking uncertain environment. Many methods [4, 14-20] have been presented for handing fuzzy multiple attributes decision-making problems. But they are only designed for standardized fuzzy numbers, that is, $\widetilde{A}=\left(a_{1}, a_{2}, a_{3}, a_{4} ; w_{\widetilde{A}}\right)$ and $0 \leq a_{1} \leq a_{2} \leq a_{3} \leq a_{4} \leq 1$. However, nonstandardized fuzzy numbers are very common in reality. Wen et al. [21] proposed a new method for ranking nonstandardized fuzzy numbers, that is, $\widetilde{A}=\left(a_{1}, a_{2}, a_{3}, a_{4} ; w_{\widetilde{A}}\right)$ and $0 \leq a_{1} \leq a_{2} \leq$ $a_{3} \leq a_{4}$. The method combines the concepts of exponential distance, the perimeter, and the area of the generalized trapezoidal fuzzy numbers for calculating the degree of similarity between nonstandardized generalized trapezoidal fuzzy numbers. But we find that the result of Wen and Zhou's method is unreasonable. Even though Wen and Zhou use exponential distance $e^{-\left|x_{\widetilde{A}}^{*}-x_{\vec{B}}^{*}\right|}$ to solve the unreasonable negative result caused by geometric distance, the problem caused by exponential distance $e^{-\left|x_{\bar{A}}^{*}-x_{\tilde{B}}^{*}\right|}$ is obvious: when $\left|x_{\widetilde{A}}^{*}-x_{\widetilde{\widetilde{B}}}^{*}\right|>1$, we always obtain unreasonable result $S(\widetilde{A}, \widetilde{B})<$ 0.37 though coincidence of the area is large and the perimeter and area of generalized trapezoidal fuzzy numbers are almost same.

Based on Wen and Zhou's method, this paper proposed a new similarity measure combines concepts of the geometric distance, the center of gravity point, the perimeter and the area of generalized trapezoidal fuzzy numbers for calculating the degree of similarity between generalized trapezoidal 
fuzzy numbers. Some properties of the proposed similarity measure have been proved, and 12 sets of generalized fuzzy numbers have been used to compare the calculation results of the proposed similarity measures with the existing similarity measures. Comparison results indicate that the proposed similarity measure can overcome the drawbacks of the existing similarity measures. Finally, a fault diagnosis experiment is carried out in laboratory based on multifunctional flexible rotor experiment bench to demonstrate the proposed similarity measure. Based on the proposed similarity measure, we present a new fuzzy fault diagnosis algorithm for dealing with rotor fault diagnosis problem, where the values of the evaluating items are represented by generalized trapezoidal fuzzy numbers. The proposed method provides us a useful way for handling fault diagnosis problem, and experimental results demonstrate that the proposed similarity measure is more effective than other methods in terms of rotor fault diagnosis.

The rest of this paper is organized as follows. In Section 2, we briefly review basic concepts of generalized fuzzy numbers. In Section 3, we briefly review some existing similarity measures of fuzzy numbers. In Section 4, we present a new similarity measure between nonstandardized generalized trapezoidal fuzzy numbers. In Section 5, we make a comparison of the calculation results of the proposed similarity measure with existing similarity measures. In Section 6, we apply the proposed similarity measure to deal with rotor fault diagnosis problem. The conclusions are discussed in Section 7 .

\section{Basic Concepts of Generalized Fuzzy Numbers}

In this section, we review basic concepts of generalized fuzzy numbers from Chen [22]. Chen represented generalized trapezoidal fuzzy number $\widetilde{A}=\left(a_{1}, a_{2}, a_{3}, a_{4}, w_{\widetilde{A}}\right)$, where $a_{1}$, $a_{2}, a_{3}$ and $a_{4}$ denote real values, $0 \leq a_{1} \leq a_{2} \leq a_{3} \leq a_{4} \leq 1$ and $0<w_{\widetilde{A}} \leq 1$, as shown in Figure 1. The generalized fuzzy number $\widetilde{A}$ is a fuzzy subset of the real line $R$. The membership function $\mu_{\widetilde{A}}$ of a generalized fuzzy number $\widetilde{A}$ satisfies the following conditions:

(1) $\mu_{\widetilde{A}}$ is a continuous mapping from the universe of discourse $R$ to the closed interval in $[0,1]$,

(2) $\mu_{\widetilde{A}}(x)=0$, where $-\infty<x \leq a_{1}$,

(3) $\mu_{\widetilde{A}}(x)$ is monotone increasing in $\left[a_{1}, a_{2}\right]$,

(4) $\mu_{\widetilde{A}}(x)=w_{\widetilde{A}}$, where $a_{2} \leq x \leq a_{3}$,

(5) $\mu_{\widetilde{A}}(x)$ is monotone decreasing in $\left[a_{3}, a_{4}\right]$,

(6) $\mu_{\widetilde{A}}(x)=0$, where $a_{4}<x \leq \infty$.

If $w_{\widetilde{A}}=1$, then the generalized fuzzy number $\widetilde{A}$ is a normal trapezoidal fuzzy number, denoted as $\widetilde{A}=$ $\left(a_{1}, a_{2}, a_{3}, a_{4}\right)$. If $a_{1}=a_{2}$ and $a_{3}=a_{4}$, then the generalized fuzzy number $\widetilde{A}$ is called a crisp interval. If $a_{1}<a_{2}=a_{3}<a_{4}$, then $\widetilde{A}$ is normal triangular fuzzy number. If $a_{1}<a_{2}<a_{3}<$ $a_{4}$, then $\widetilde{A}$ is a generalized trapezoidal fuzzy number. If $a_{1}=$ $a_{2}=a_{3}=a_{4}$ and $w_{\widetilde{A}}=1$, then $\widetilde{A}$ is a real number.

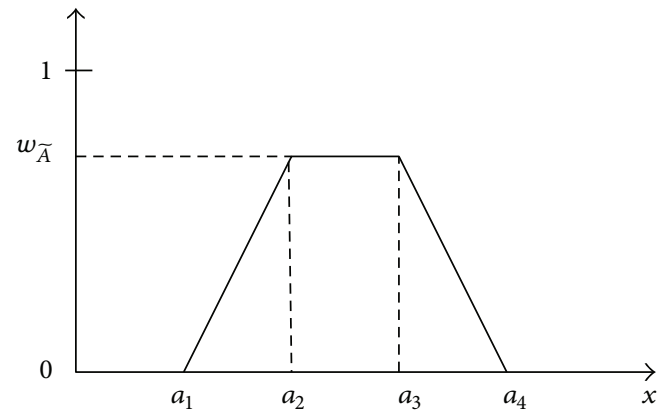

FIGURE 1: A generalized trapezoidal fuzzy number.

\section{Existing Similarity Measure between Fuzzy Numbers}

In this section, some works have to be done to review some existing similarity measures between fuzzy numbers from Chen [14], Lee [15], S.-J. Chen and S.-M. Chen [4], Wei and Chen [20], and Wen et al. [21]. Consider two trapezoidal fuzzy numbers $\widetilde{A}$ and $\widetilde{B}$, where $\widetilde{A}=\left(a_{1}, a_{2}, a_{3}, a_{4}, w_{\widetilde{A}}\right)$ and $\widetilde{B}=\left(b_{1}, b_{2}, b_{3}, b_{4}, w_{\widetilde{B}}\right)$.

3.1. Chen's Similarity Measures between Fuzzy Numbers. Chen [14] presented a distance-based similarity measure between two trapezoidal fuzzy numbers $\widetilde{A}$ and $\widetilde{B}$, where $\widetilde{A}=$ $\left(a_{1}, a_{2}, a_{3}, a_{4}\right)$ and $\widetilde{B}=\left(b_{1}, b_{2}, b_{3}, b_{4}\right)$. The degree of similarity $S(\widetilde{A}, \widetilde{B})$ between $\widetilde{A}$ and $\widetilde{B}$ is calculated as follows:

$$
S(\widetilde{A}, \widetilde{B})=1-\frac{\sum_{i=1}^{4}\left|a_{i}-b_{i}\right|}{4},
$$

where $S(\widetilde{A}, \widetilde{B}) \in[0,1]$, if $\widetilde{A}$ and $\widetilde{B}$ are triangular fuzzy numbers, where $\widetilde{A}=\left(a_{1}, a_{2}, a_{3}\right)$ and $\widetilde{B}=\left(b_{1}, b_{2}, b_{3}\right)$. The degree of similarity $S(\widetilde{A}, \widetilde{B})$ between $\widetilde{A}$ and $\widetilde{B}$ is calculated as follows:

$$
S(\widetilde{A}, \widetilde{B})=1-\frac{\sum_{i=1}^{3}\left|a_{i}-b_{i}\right|}{3} .
$$

The larger the value of $S(\widetilde{A}, \widetilde{B})$, the more similarity between the fuzzy numbers $\widetilde{A}$ and $\widetilde{B}$.

3.2. Lee's Similarity Measures between Fuzzy Numbers. Lee [15] presented a similarity measure between two trapezoidal fuzzy numbers $\widetilde{A}$ and $\widetilde{B}$, where $\widetilde{A}=\left(a_{1}, a_{2}, a_{3}, a_{4}\right)$ and $\widetilde{B}=$ $\left(b_{1}, b_{2}, b_{3}, b_{4}\right)$. The degree of similarity $S(\widetilde{A}, \widetilde{B})$ between $\widetilde{A}$ and $\widetilde{B}$ is calculated as follows:

$$
S(\widetilde{A}, \widetilde{B})=1-\frac{\|\widetilde{A}-\widetilde{B}\|_{l p}}{\|U\|} \times 4^{-1 / p}
$$

where

$$
\begin{gathered}
\|\widetilde{A}-\widetilde{B}\|_{l p}=\left(\sum_{i=1}^{4}\left(\left|a_{i}-b_{i}\right|\right)^{p}\right)^{1 / p}, \\
\|U\|=\max (U)-\min (U) .
\end{gathered}
$$


3.3. Chen and Chen's Similarity Measures between Fuzzy Numbers. Chen's and Lee's similarity measures cannot deal with generalized fuzzy numbers. In order to solve this problem, S.-J. Chen and S.-M. Chen [4] presented a similarity measure between two generalized trapezoidal fuzzy numbers $\widetilde{A}=\left(a_{1}, a_{2}, a_{3}, a_{4} ; w_{\widetilde{A}}\right)$ and $\widetilde{B}=\left(b_{1}, b_{2}, b_{3}, b_{4} ; w_{\widetilde{B}}\right)$ by using the simple center of gravity method (SCGM). The degree of similarity $S(\widetilde{A}, \widetilde{B})$ between $\widetilde{A}$ and $\widetilde{B}$ is calculated as follows:

$$
\begin{aligned}
S(\widetilde{A}, \widetilde{B})= & {\left[1-\frac{\sum_{i=1}^{4}\left|a_{i}-b_{i}\right|}{4}\right] \times\left(1-\left|x_{\widetilde{A}}^{*}-x_{\widetilde{B}}^{*}\right|\right)^{B\left(S_{\widetilde{A}}, S_{\widetilde{B}}\right)} } \\
& \times \frac{\min \left(y_{\widetilde{A}}^{*}, y_{\widetilde{B}}^{*}\right)}{\max \left(y_{\widetilde{A}}^{*}, y_{\widetilde{B}}^{*}\right)}
\end{aligned}
$$

where $S(\widetilde{A}, \widetilde{B}) \in[0,1]$, and $B\left(S_{\widetilde{A}}, S_{\widetilde{B}}\right)$ is defined as follows:

$$
B\left(S_{\widetilde{A}}, S_{\widetilde{B}}\right)= \begin{cases}1, & \text { if } S_{\widetilde{A}}+S_{\widetilde{B}}>0, \\ 0, & \text { if } S_{\widetilde{A}}+S_{\widetilde{B}}=0\end{cases}
$$

where $S_{\widetilde{A}}$ and $S_{\widetilde{B}}$ are defined as follows:

$$
\begin{gathered}
S_{\widetilde{A}}=a_{4}-a_{1}, \\
S_{\widetilde{B}}=b_{4}-b_{1} .
\end{gathered}
$$

Then, the center of gravity (COG) point $\left(x_{\widetilde{A}}^{*}, y_{\widetilde{A}}^{*}\right)$ and $\left(x_{\widetilde{B}}^{*}, y_{\widetilde{B}}^{*}\right)$ of generalized trapezoidal fuzzy numbers $\widetilde{A}$ and $\widetilde{B}$ can be calculated as follows:

$$
\begin{gathered}
y_{\widetilde{A}}^{*}=\left\{\begin{array}{lc}
\frac{w_{\widetilde{A}} \times\left(\left(a_{3}-a_{2}\right) /\left(a_{4}-a_{1}\right)+2\right)}{6}, & \text { if } a_{4} \neq a_{1}, \\
\frac{w_{\widetilde{A}}}{2}, & 0<w_{\widetilde{A}} \leq 1
\end{array}\right. \\
x_{\widetilde{A}}^{*}=\frac{y_{\widetilde{A}}^{*}\left(a_{3}+a_{2}\right)+\left(a_{4}+a_{1}\right)\left(w_{\widetilde{A}}-y_{\widetilde{A}}^{*}\right)}{2 w_{\widetilde{A}}} . \\
\end{gathered}
$$

The values of $x_{\widetilde{B}}^{*}$ and $y_{\widetilde{B}}^{*}$ can be calculated as the same.

3.4. Wei and Chen's Similarity Measures between Fuzzy Numbers. Wei and Chen [20] presented a new method by combining concepts of the geometric distance, the perimeter, and the height of generalized fuzzy numbers to calculate the degree of similarity $S(\widetilde{A}, \widetilde{B})$ between $\widetilde{A}$ and $\widetilde{B}$, where $\widetilde{A}=$ $\left(a_{1}, a_{2}, a_{3}, a_{4} ; w_{\widetilde{A}}\right), \widetilde{B}=\left(b_{1}, b_{2}, b_{3}, b_{4} ; w_{\widetilde{B}}\right), 0 \leq a_{1} \leq a_{2} \leq a_{3} \leq$ $a_{4} \leq 1$, and $0 \leq b_{1} \leq b_{2} \leq b_{3} \leq b_{4} \leq 1$. The degree of similarity $S(\widetilde{A}, \widetilde{B})$ between $\widetilde{A}$ and $\widetilde{B}$ is calculated as follows:

$$
\begin{aligned}
S(\widetilde{A}, \widetilde{B})= & {\left[1-\frac{\sum_{i=1}^{4}\left|a_{i}-b_{i}\right|}{4}\right] } \\
& \times \frac{\min (P(\widetilde{A}), P(\widetilde{B}))+\min \left(w_{\widetilde{A}}, w_{\widetilde{B}}\right)}{\max (P(\widetilde{A}), P(\widetilde{B}))+\max \left(w_{\widetilde{A}}, w_{\widetilde{B}}\right)},
\end{aligned}
$$

where $S(\widetilde{A}, \widetilde{B}) \in[0,1]$, and the perimeters $P(\widetilde{A})$ and $P(\widetilde{B})$ are defined as follows:

$$
\begin{aligned}
P(\widetilde{A})= & \sqrt{\left(a_{1}-a_{2}\right)^{2}+w_{\widetilde{A}}^{2}}+\sqrt{\left(a_{3}-a_{4}\right)^{2}+w_{\widetilde{A}}^{2}} \\
& +\left(a_{3}-a_{2}\right)+\left(a_{4}-a_{1}\right), \\
P(\widetilde{B})= & \sqrt{\left(b_{1}-b_{2}\right)^{2}+w_{\widetilde{B}}^{2}}+\sqrt{\left(b_{3}-b_{4}\right)^{2}+w_{\widetilde{B}}^{2}} \\
& +\left(b_{3}-b_{2}\right)+\left(b_{4}-b_{1}\right) .
\end{aligned}
$$

3.5. Wen and Zhou's Similarity Measures between Fuzzy Numbers. All of former four similarity measures are only designed for standardized fuzzy numbers, that is, $\widetilde{A}=$ $\left(a_{1}, a_{2}, a_{3}, a_{4} ; w_{\widetilde{A}}\right)$ and $0 \leq a_{1} \leq a_{2} \leq a_{3} \leq a_{4} \leq 1$. They cannot be used to calculate the degree of similarity between nonstandardized fuzzy numbers (i.e., $\widetilde{A}=\left(a_{1}, a_{2}, a_{3}, a_{4} ; w_{\widetilde{A}}\right)$ and $\left.0 \leq a_{1} \leq a_{2} \leq a_{3} \leq a_{4}\right)$. This is because they all use geometric distance $\sum_{i=1}^{4}\left|a_{i}-b_{i}\right| / 4$ to measure the degree of similarity $S(\widetilde{A}, \widetilde{B})$, which may lead to unreasonable negative result easily, that is, $S(\widetilde{A}, \widetilde{B})<0$. However, nonstandardized fuzzy numbers are very common in reality. Therefore, it is necessary to put forward a new similarity measure. In order to deal with both standardized and nonstandardized fuzzy numbers, Wen et al. [21] put forward a new method combines concepts of the exponential distance, the perimeter, and the area of the generalized trapezoidal fuzzy numbers for calculating the degree of similarity $S(\widetilde{A}, \widetilde{B})$ between $\widetilde{A}$ and $\widetilde{B}$, where $\widetilde{A}=\left(a_{1}, a_{2}, a_{3}, a_{4} ; w_{\widetilde{A}}\right), \widetilde{B}=\left(b_{1}, b_{2}, b_{3}, b_{4} ; w_{\widetilde{B}}\right)$, $0 \leq a_{1} \leq a_{2} \leq a_{3} \leq a_{4}$, and $0 \leq b_{1} \leq b_{2} \leq b_{3} \leq b_{4}$. The degree of similarity $S(\widetilde{A}, \widetilde{B})$ between $\widetilde{A}$ and $\widetilde{B}$ is calculated as follows:

$$
\begin{aligned}
S(\widetilde{A}, \widetilde{B})= & e^{-\left|x_{\widetilde{A}}^{*}-x_{\widetilde{B}}^{*}\right|} \\
& \times \frac{\min (P(\widetilde{A}), P(\widetilde{B}))+\min (a(\widetilde{A}), a(\widetilde{B}))}{\max (P(\widetilde{A}), P(\widetilde{B}))+\max (a(\widetilde{A}), a(\widetilde{B}))},
\end{aligned}
$$

where $S(\widetilde{A}, \widetilde{B}) \in[0,1]$, the center of gravity point $\left(x_{\widetilde{A}}^{*}, y_{\widetilde{A}}^{*}\right)$ is calculated as (8), and the perimeters $P(\widetilde{A})$ and the area $a(\widetilde{A})$ of generalized trapezoidal fuzzy number $\widetilde{A}$ are calculated as follows:

$$
\begin{gathered}
P(\widetilde{A})= \begin{cases}\sqrt{\left(a_{1}-a_{2}\right)^{2}+w_{\widetilde{A}}^{2}}+\sqrt{\left(a_{3}-a_{4}\right)^{2}+w_{\widetilde{A}}^{2}} \\
+\left(a_{3}-a_{2}\right)+\left(a_{4}-a_{1}\right), & \text { if } a_{4}-a_{1} \neq 0 \\
w_{\widetilde{A}}, & \text { if } a_{4}-a_{1}=0\end{cases} \\
a(\widetilde{A})=\frac{1}{2} \times\left[\left(a_{2}-a_{1}\right)+\left(a_{4}-a_{3}\right)\right] \times w_{\widetilde{A}}+\left(a_{3}-a_{2}\right) \times w_{\widetilde{A}} .
\end{gathered}
$$

The center of gravity (COG) point $\left(x_{\widetilde{B}}^{*}, y_{\widetilde{B}}^{*}\right)$, the perimeter $P(\widetilde{B})$, and the area $a(\widetilde{B})$ of generalized trapezoidal fuzzy number $\widetilde{B}$ can be calculated as the same.

Even though Wen and Zhou use exponential distance $e^{-\left|x_{\widetilde{A}}^{*}-x_{\tilde{B}}^{*}\right|}$ to solve the unreasonable negative result caused 
by geometric distance $\sum_{i=1}^{4}\left|a_{i}-b_{i}\right| / 4$, the problem caused by exponential distance $e^{-\left|x_{\widetilde{A}}^{*}-x_{\widetilde{B}}^{*}\right|}$ is obvious: the effect of exponential distance on $S(\widetilde{A}, \widetilde{B})$ is more than the perimeter and the area of the generalized trapezoidal fuzzy numbers. When $\left|x_{\widetilde{A}}^{*}-x_{\widetilde{B}}^{*}\right|>1$, we always obtain the unreasonable result $S(\widetilde{A}, \widetilde{B})<0.37$, though the coincidence of the area is large and the perimeter and area of generalized trapezoidal fuzzy numbers are almost the same. Therefore, it is necessary to put forward a new similarity measure to solve the existing defects of Wen and Zhou's similarity measure.

\section{A New Similarity Measure between Generalized Trapezoidal Fuzzy Numbers}

In this section, a new method is presented to calculate the degree of similarity between generalized fuzzy numbers based on Wen and Zhou's method. The proposed method combines concepts of the geometric distance, the center of gravity point, the perimeter, and the area of the generalized trapezoidal fuzzy numbers for calculating the degree of similarity $S(\widetilde{A}, \widetilde{B})$ between $\widetilde{A}$ and $\widetilde{B}$, where $\widetilde{A}=\left(a_{1}, a_{2}, a_{3}, a_{4} ; w_{\widetilde{A}}\right)$, $\widetilde{B}=\left(b_{1}, b_{2}, b_{3}, b_{4} ; w_{\widetilde{B}}\right), 0 \leq a_{1} \leq a_{2} \leq a_{3} \leq a_{4}, 0 \leq b_{1} \leq$ $b_{2} \leq b_{3} \leq b_{4}, 0 \leq w_{\widetilde{A}} \leq 1$, and $0 \leq w_{\widetilde{B}} \leq 1$. The proposed method not only can deal with standardized fuzzy numbers but also can deal with nonstandardized fuzzy numbers. In order to deal with nonstandardized fuzzy numbers, normalization process is used to transform it into standardized fuzzy numbers which helps to avoid obtaining unreasonable negative result $S(\widetilde{A}, \widetilde{B})<0$. Normalization process is shown as follows:

$$
\begin{aligned}
& a_{1}^{\prime}=\frac{a_{1}-\min \left(a_{1}, b_{1}\right)}{\max \left(a_{4}, b_{4}\right)-\min \left(a_{1}, b_{1}\right)}, \\
& a_{2}^{\prime}=\frac{a_{2}-\min \left(a_{1}, b_{1}\right)}{\max \left(a_{4}, b_{4}\right)-\min \left(a_{1}, b_{1}\right)}, \\
& a_{3}^{\prime}=\frac{a_{3}-\min \left(a_{1}, b_{1}\right)}{\max \left(a_{4}, b_{4}\right)-\min \left(a_{1}, b_{1}\right)}, \\
& a_{4}^{\prime}=\frac{a_{4}-\min \left(a_{1}, b_{1}\right)}{\max \left(a_{4}, b_{4}\right)-\min \left(a_{1}, b_{1}\right)} .
\end{aligned}
$$

Then, the new standardized fuzzy number $\widetilde{A^{\prime}}$ can be got, where $\widetilde{A^{\prime}}=\left(a_{1}^{\prime}, a_{2}^{\prime}, a_{3}^{\prime}, a_{4}^{\prime} ; w_{\widetilde{A}}\right)$ and $0 \leq a_{1}^{\prime} \leq a_{2}^{\prime} \leq a_{3}^{\prime} \leq a_{4}^{\prime} \leq 1$. The new standardized fuzzy number $\widetilde{B^{\prime}}$ can be got in the same way, where $\widetilde{B^{\prime}}=\left(b_{1}^{\prime}, b_{2}^{\prime}, b_{3}^{\prime}, b_{4}^{\prime} ; w_{\widetilde{B}}\right)$ and $0 \leq b_{1}^{\prime} \leq b_{2}^{\prime} \leq b_{3}^{\prime} \leq$ $b_{4}^{\prime} \leq 1$. The degree of similarity $S(\widetilde{A}, \widetilde{B})$ between $\widetilde{A}$ and $\widetilde{B}$ is calculated as follows:

$$
\begin{aligned}
S(\widetilde{A}, \widetilde{B})= & S\left(\widetilde{A^{\prime}}, \widetilde{B^{\prime}}\right)=\left[1-\frac{\sum_{i=1}^{4}\left|a_{i}^{\prime}-b_{i}^{\prime}\right|}{4}\right]^{\left(1-\left|x_{\widetilde{A^{\prime}}}^{*}-x_{\bar{B}^{\prime}}^{*}\right|\right)} \\
& \times \frac{\min \left(P\left(\widetilde{A^{\prime}}\right), P\left(\widetilde{B^{\prime}}\right)\right)+\min \left(a\left(\widetilde{A^{\prime}}\right), a\left(\widetilde{B^{\prime}}\right)\right)}{\max \left(P\left(\widetilde{A^{\prime}}\right), P\left(\widetilde{B^{\prime}}\right)\right)+\max \left(a\left(\widetilde{A^{\prime}}\right), a\left(\widetilde{B^{\prime}}\right)\right)} .
\end{aligned}
$$

The normalization process is unworkable when the generalized trapezoidal fuzzy numbers $\widetilde{A}$ and $\widetilde{B}$ are real numbers at the same time, where $\widetilde{A}=\left(a_{1}, a_{2}, a_{3}, a_{4} ; w_{\widetilde{A}}\right), \widetilde{B}=$ $\left(b_{1}, b_{2}, b_{3}, b_{4} ; w_{\widetilde{B}}\right), a_{1}=a_{2}=a_{3}=a_{4}, b_{1}=b_{2}=b_{3}=b_{4}$, $0 \leq w_{\widetilde{A}} \leq 1$, and $0 \leq w_{\widetilde{B}} \leq 1$. In order to deal with this problem, the geometric distance should be transformed into exponential distance. The degree of similarity $S(\widetilde{A}, \widetilde{B})$ between $\widetilde{A}$ and $\widetilde{B}$ is calculated as follows:

$$
\begin{aligned}
S(\widetilde{A}, \widetilde{B})= & e^{-\left|\left(\sum_{i=1}^{4}\left|a_{i}-b_{i}\right| / 4\right) \times\left(1-\left|x_{\widetilde{A}}^{*}-x_{\widetilde{B}}^{*}\right|\right)\right|} \\
& \times \frac{\min (P(\widetilde{A}), P(\widetilde{B}))+\min (a(\widetilde{A}), a(\widetilde{B}))}{\max (P(\widetilde{A}), P(\widetilde{B}))+\max (a(\widetilde{A}), a(\widetilde{B}))},
\end{aligned}
$$

where $S(\widetilde{A}, \widetilde{B}) \in[0,1]$, and the larger the value of $S(\widetilde{A}, \widetilde{B})$, the more similarity between the fuzzy numbers $\widetilde{A}$ and $\widetilde{B}$.

4.1. Properties of the New Similarity Measure. We can verify the following properties of the new similarity measure.

Property 1. $S(\widetilde{A}, \widetilde{B}) \in[0,1]$.

Proof. When the generalized trapezoidal fuzzy numbers $\widetilde{A}$ and $\widetilde{B}$ are not real numbers at the same time, and because (1$\left.\left(\sum_{i=1}^{4}\left|a_{i}^{\prime}-b_{i}^{\prime}\right| / 4\right)\right) \in[0,1],\left(1-\left|x_{\widetilde{A^{\prime}}}^{*}-x_{\widetilde{B^{\prime}}}^{*}\right|\right) \in[0,1], \min \left(P\left(\widetilde{A^{\prime}}\right)\right.$, $\left.P\left(\widetilde{B^{\prime}}\right)\right) \leq \max \left(P\left(\widetilde{A^{\prime}}\right), P\left(\widetilde{B^{\prime}}\right)\right)$, and $\min \left(a\left(\widetilde{A^{\prime}}\right), a\left(\widetilde{B^{\prime}}\right)\right) \leq$ $\max \left(a\left(\widetilde{A^{\prime}}\right), a\left(\widetilde{B^{\prime}}\right)\right)$, we can obtain $S(\widetilde{A}, \widetilde{B}) \in[0,1]$.

When the generalized trapezoidal fuzzy numbers $\widetilde{A}$ and $\widetilde{B}$ are real numbers at the same time, and because $e^{-\left|\left(\sum_{i=1}^{4}\left|a_{i}-b_{i}\right| / 4\right) \times\left(1-\left|x_{\widetilde{A}}^{*}-x_{\widetilde{B}}^{*}\right|\right)\right|} \in[0,1], \min (P(\widetilde{A}), P(\widetilde{B})) \leq$ $\max (P(\widetilde{A}), P(\widetilde{B}))$, and $\min (a(\widetilde{A}), a(\widetilde{B})) \leq \max (a(\widetilde{A}), a(\widetilde{B}))$, we can obtain $S(\widetilde{A}, \widetilde{B}) \in[0,1]$.

The proof is completed.

Property 2. Two generalized trapezoidal fuzzy numbers $\widetilde{A}$ and $\widetilde{B}$ are identical if and only if $S(\widetilde{A}, \widetilde{B})=1$.

Proof. When the generalized trapezoidal fuzzy numbers $\widetilde{A}$ and $\widetilde{B}$ are not real numbers at the same time, if $\widetilde{A}$ and $\widetilde{B}$ are identical, then $a_{1}=b_{1}, a_{2}=b_{2}, a_{3}=b_{3}, a_{4}=b_{4}$ and $w_{\widetilde{A}}=w_{\widetilde{B}}$. Thus, $a_{1}^{\prime}=b_{1}^{\prime}, a_{2}^{\prime}=b_{2}^{\prime}, a_{3}^{\prime}=b_{3}^{\prime}, a_{4}^{\prime}=b_{4}^{\prime}, \min \left(P\left(\widetilde{A^{\prime}}\right), P\left(\widetilde{B^{\prime}}\right)\right)=$ $\max \left(P\left(\widetilde{A^{\prime}}\right), P\left(\widetilde{B^{\prime}}\right)\right), \min \left(a\left(\widetilde{A^{\prime}}\right), a\left(\widetilde{B^{\prime}}\right)\right)=\max \left(a\left(\widetilde{A^{\prime}}\right), a\left(\widetilde{B^{\prime}}\right)\right)$, and $x_{\widetilde{A^{\prime}}}^{*}=x_{\widetilde{B^{\prime}}}^{*}$. The degree of similarity $S(\widetilde{A}, \widetilde{B})$ between $\widetilde{A}$ and $\widetilde{B}$ is calculated as follows:

$$
\begin{aligned}
S(\widetilde{A}, \widetilde{B})= & S\left(\widetilde{A^{\prime}}, \widetilde{B^{\prime}}\right)=\left[1-\frac{\sum_{i=1}^{4}\left|a_{i}^{\prime}-b_{i}^{\prime}\right|}{4}\right]^{\left(1-\left|x_{\widetilde{A^{\prime}}}^{*}-x_{B^{\prime}}^{*}\right|\right)} \\
& \times \frac{\min \left(P\left(\widetilde{A^{\prime}}\right), P\left(\widetilde{B^{\prime}}\right)\right)+\min \left(a\left(\widetilde{A^{\prime}}\right), a\left(\widetilde{B^{\prime}}\right)\right)}{\max \left(P\left(\widetilde{A^{\prime}}\right), P\left(\widetilde{B^{\prime}}\right)\right)+\max \left(a\left(\widetilde{A^{\prime}}\right), a\left(\widetilde{B^{\prime}}\right)\right)} \\
= & (1-0)^{1} \times 1=1 .
\end{aligned}
$$


When the generalized trapezoidal fuzzy numbers $\widetilde{A}$ and $\widetilde{B}$ are real numbers at the same time, if $\widetilde{A}$ and $\widetilde{B}$ are identical, thus, $a_{1}=a_{2}=a_{3}=a_{4}=b_{1}=b_{2}=b_{3}=b_{4}, \min (P(\widetilde{A}), P(\widetilde{B}))=$ $\max (P(\widetilde{A}), P(\widetilde{B})), \min (a(\widetilde{A}), a(\widetilde{B}))=\max (a(\widetilde{A}), a(\widetilde{B}))$, and $x_{\widetilde{A}}^{*}=x_{\widetilde{B}}^{*}$. The degree of similarity $\mathrm{S}(\widetilde{A}, \widetilde{B})$ between $\widetilde{A}$ and $\widetilde{B}$ is calculated as follows:

$$
\begin{aligned}
S(\widetilde{A}, \widetilde{B})= & e^{-\left|\left(\sum_{i=1}^{4}\left|a_{i}-b_{i}\right| / 4\right) \times\left(1-\left|x_{\widetilde{A}}^{*}-x_{\widetilde{B}}^{*}\right|\right)\right|} \\
& \times \frac{\min (P(\widetilde{A}), P(\widetilde{B}))+\min (a(\widetilde{A}), a(\widetilde{B}))}{\max (P(\widetilde{A}), P(\widetilde{B}))+\max (a(\widetilde{A}), a(\widetilde{B}))} \\
= & e^{-|0 \times 1|}=1 .
\end{aligned}
$$

When the generalized trapezoidal fuzzy numbers $\widetilde{A}$ and $\widetilde{B}$ are not real numbers at the same time, if $S(\widetilde{A}, \widetilde{B})=S\left(\widetilde{A^{\prime}}, \widetilde{B^{\prime}}\right)=1$, then

$$
\begin{aligned}
S(\widetilde{A}, \widetilde{B})= & S\left(\widetilde{A^{\prime}}, \widetilde{B^{\prime}}\right)=\left[1-\frac{\sum_{i=1}^{4}\left|a_{i}^{\prime}-b_{i}^{\prime}\right|}{4}\right]^{\left(1-\mid x_{\widetilde{A^{\prime}}}^{*}-x_{\widetilde{B^{\prime}}}^{*}, 1\right)} \\
& \times \frac{\min \left(P\left(\widetilde{A^{\prime}}\right), P\left(\widetilde{B^{\prime}}\right)\right)+\min \left(a\left(\widetilde{A^{\prime}}\right), a\left(\widetilde{B^{\prime}}\right)\right)}{\max \left(P\left(\widetilde{A^{\prime}}\right), P\left(\widetilde{B^{\prime}}\right)\right)+\max \left(a\left(\widetilde{A^{\prime}}\right), a\left(\widetilde{B^{\prime}}\right)\right)}
\end{aligned}
$$$$
=1 \text {. }
$$

It implies that $a_{1}^{\prime}=b_{1}^{\prime}, a_{2}^{\prime}=b_{2}^{\prime}, a_{3}^{\prime}=b_{3}^{\prime}, a_{4}^{\prime}=b_{4}^{\prime}$, $\min \left(P\left(\widetilde{A^{\prime}}\right), P\left(\widetilde{B^{\prime}}\right)\right)=\max \left(P\left(\widetilde{A^{\prime}}\right), P\left(\widetilde{B^{\prime}}\right)\right), \min \left(a\left(\widetilde{A^{\prime}}\right), a\left(\widetilde{B^{\prime}}\right)\right)=$ $\max \left(a\left(\widetilde{A^{\prime}}\right), a\left(\widetilde{B^{\prime}}\right)\right)$, and $x_{\widetilde{A^{\prime}}}^{*}=x_{\widetilde{B^{\prime}}}^{*}$. Therefore, $a_{1}=b_{1}, a_{2}=$ $b_{2}, a_{3}=b_{3}, a_{4}=b_{4}$, and $w_{\widetilde{A}}=w_{\widetilde{B}}$, the generalized trapezoidal fuzzy numbers $\widetilde{A}$ and $\widetilde{B}$ are identical.

When the generalized trapezoidal fuzzy numbers $\widetilde{A}$ and $\widetilde{B}$ are real numbers at the same time, if $S(\widetilde{A}, \widetilde{B})=S\left(\widetilde{A^{\prime}}, \widetilde{B^{\prime}}\right)=1$, then

$$
\begin{aligned}
S(\widetilde{A}, \widetilde{B})= & e^{-\left|\left(\sum_{i=1}^{4}\left|a_{i}-b_{i}\right| / 4\right) \times\left(1-\left|x_{\widetilde{A}}^{*}-x_{\widetilde{B}}^{*}\right|\right)\right|} \\
& \times \frac{\min (P(\widetilde{A}), P(\widetilde{B}))+\min (a(\widetilde{A}), a(\widetilde{B}))}{\max (P(\widetilde{A}), P(\widetilde{B}))+\max (a(\widetilde{A}), a(\widetilde{B}))}=1 .
\end{aligned}
$$

It implies that $a_{1}=b_{1}, a_{2}=b_{2}, a_{3}=b_{3}, a_{4}=b_{4}$, $\min (P(\widetilde{A}), P(\widetilde{B}))=\max (P(\widetilde{A}), P(\widetilde{B})), \min (a(\widetilde{A}), a(\widetilde{B}))=$ $\max (a(\widetilde{A}), a(\widetilde{B}))$, and $x_{\widetilde{A}}^{*}=x_{\widetilde{B}}^{*}$. Therefore, $a_{1}=b_{1}, a_{2}=$ $b_{2}, a_{3}=b_{3}, a_{4}=b_{4}$, and $w_{\widetilde{A}}=w_{\widetilde{B}}$, the generalized trapezoidal fuzzy numbers $\widetilde{A}$ and $\widetilde{B}$ are identical.

The proof is completed.

Property 3. $S(\widetilde{A}, \widetilde{B})=S(\widetilde{B}, \widetilde{A})$.
Proof. When the generalized trapezoidal fuzzy numbers $\widetilde{A}$ and $\widetilde{B}$ are not real numbers at the same time,

$$
\begin{aligned}
S(\widetilde{A}, \widetilde{B})= & S\left(\widetilde{A^{\prime}}, \widetilde{B^{\prime}}\right)=\left[1-\frac{\sum_{i=1}^{4}\left|a_{i}^{\prime}-b_{i}^{\prime}\right|}{4}\right]^{\left(1-\left|x_{A^{\prime}}^{*}-x_{\widetilde{B^{\prime}}}^{*}\right|\right)} \\
& \times \frac{\min \left(P\left(\widetilde{A^{\prime}}\right), P\left(\widetilde{B^{\prime}}\right)\right)+\min \left(a\left(\widetilde{A^{\prime}}\right), a\left(\widetilde{B^{\prime}}\right)\right)}{\max \left(P\left(\widetilde{A^{\prime}}\right), P\left(\widetilde{B^{\prime}}\right)\right)+\max \left(a\left(\widetilde{A^{\prime}}\right), a\left(\widetilde{B^{\prime}}\right)\right)}, \\
S(\widetilde{A}, \widetilde{B})= & S\left(\widetilde{B^{\prime}}, \widetilde{A^{\prime}}\right)=\left[1-\frac{\sum_{i=1}^{4}\left|b_{i}^{\prime}-a_{i}^{\prime}\right|}{4}\right]^{\left(1-\left|x_{B^{\prime}}^{*}-x_{\widetilde{A^{\prime}}}^{*}\right|\right)} \\
& \times \frac{\min \left(P\left(\widetilde{B^{\prime}}\right), P\left(\widetilde{A^{\prime}}\right)\right)+\min \left(a\left(\widetilde{B^{\prime}}\right), a\left(\widetilde{A^{\prime}}\right)\right)}{\max \left(P\left(\widetilde{B^{\prime}}\right), P\left(\widetilde{A^{\prime}}\right)\right)+\max \left(a\left(\widetilde{B^{\prime}}\right), a\left(\widetilde{A^{\prime}}\right)\right)} .
\end{aligned}
$$

Because $\sum_{i=1}^{4}\left|a_{i}^{\prime}-b_{i}^{\prime}\right|=\sum_{i=1}^{4}\left|b_{i}^{\prime}-a_{i}^{\prime}\right|$, based on (12), we can obtain that $\min \left(P\left(\widetilde{A^{\prime}}\right), P\left(\widetilde{B^{\prime}}\right)\right)=\min \left(P\left(\widetilde{B^{\prime}}\right), P\left(\widetilde{A^{\prime}}\right)\right), \max \left(P\left(\widetilde{A^{\prime}}\right)\right.$, $\left.P\left(\widetilde{B^{\prime}}\right)\right)=\max \left(P\left(\widetilde{B^{\prime}}\right), P\left(\widetilde{A^{\prime}}\right)\right), \min \left(a\left(\widetilde{A^{\prime}}\right), a\left(\widetilde{B^{\prime}}\right)\right)=\min \left(a\left(\widetilde{B^{\prime}}\right)\right.$, $\left.a\left(\widetilde{A^{\prime}}\right)\right), \max \left(a\left(\widetilde{A^{\prime}}\right), a\left(\widetilde{B^{\prime}}\right)\right)=\max \left(a\left(\widetilde{B^{\prime}}\right), a\left(\widetilde{A^{\prime}}\right)\right)$, and $\mid x_{\widetilde{A^{\prime}}}^{*}-$ $x_{\widetilde{B^{\prime}}}^{*}|=| x_{\widetilde{B^{\prime}}}^{*}-x_{\widetilde{A^{\prime}}}^{*} \mid$. Therefore, $S(\widetilde{A}, \widetilde{B})=S(\widetilde{B}, \widetilde{A})$.

When the generalized trapezoidal fuzzy numbers $\widetilde{A}$ and $\widetilde{B}$ are real numbers at the same time,

$$
\begin{aligned}
S(\widetilde{A}, \widetilde{B})= & e^{-\left|\left(\sum_{i=1}^{4}\left|a_{i}-b_{i}\right| / 4\right) \times\left(1-\left|x_{\widetilde{A}}^{*}-x_{\widetilde{B}}^{*}\right|\right)\right|} \\
& \times \frac{\min (P(\widetilde{A}), P(\widetilde{B}))+\min (a(\widetilde{A}), a(\widetilde{B}))}{\max (P(\widetilde{A}), P(\widetilde{B}))+\max (a(\widetilde{A}), a(\widetilde{B}))}, \\
S(\widetilde{B}, \widetilde{A})= & e^{-\left|\left(\sum_{i=1}^{4}\left|b_{i}-a_{i}\right| / 4\right) \times\left(1-\left|x_{\widetilde{B}}^{*}-x_{\widetilde{A}}^{*}\right|\right)\right|} \\
& \times \frac{\min (P(\widetilde{B}), P(\widetilde{A}))+\min (a(\widetilde{B}), a(\widetilde{A}))}{\max (P(\widetilde{B}), P(\widetilde{A}))+\max (a(\widetilde{B}), a(\widetilde{A}))} .
\end{aligned}
$$

Because $\sum_{i=1}^{4}\left|a_{i}-b_{i}\right|=\sum_{i=1}^{4}\left|b_{i}-a_{i}\right|$, based on (12), we can obtain that $\min (P(\widetilde{A}), P(\widetilde{B}))=\min (P(\widetilde{B}), P(\widetilde{A})), \max (P(\widetilde{A})$, $P(\widetilde{B}))=\max (P(\widetilde{B}), P(\widetilde{A})), \min (a(\widetilde{A}), a(\widetilde{B}))=\min (a(\widetilde{B})$, $a(\widetilde{A})), \max (a(\widetilde{A}), a(\widetilde{B}))=\max (a(\widetilde{B}), a(\widetilde{A}))$, and $\left|x_{\widetilde{A}}^{*}-x_{\widetilde{B}}^{*}\right|=$ $\left|x_{\widetilde{B}}^{*}-x_{\widetilde{A}}^{*}\right|$. Therefore, $S(\widetilde{A}, \widetilde{B})=S(\widetilde{B}, \widetilde{A})$.

The proof is completed.

\section{The Comparison of the Similarity Measures}

In this section, the proposed similarity measure is compared with five existing methods (Chen's method, Lee's method, Chen and Chen's method, Wei and Chen's method, and Wen and Zhou's method) using 12 sets of generalized fuzzy numbers shown in Figure 2. Set 1 to set 8 are 8 sets of standardized generalized fuzzy numbers, and set 9 to set 12 are 4 sets of nonstandardized generalized fuzzy numbers. Table 1 compares the calculation results of all six similarity measures. 


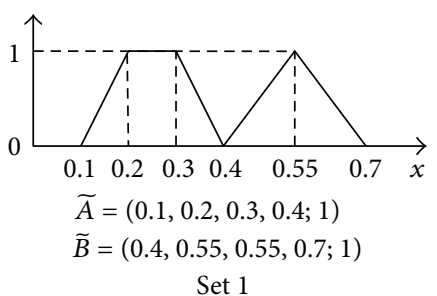

Set 1

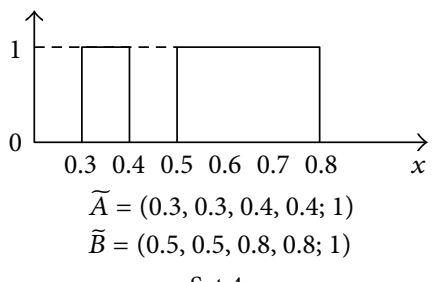

Set 4

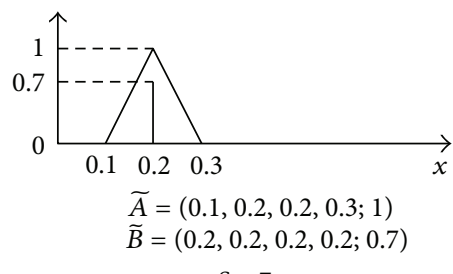

Set 7

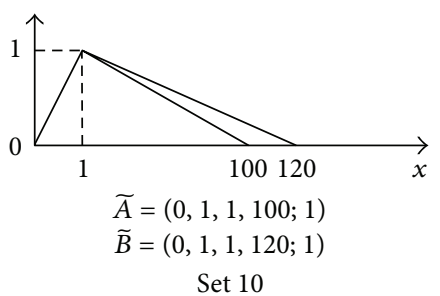

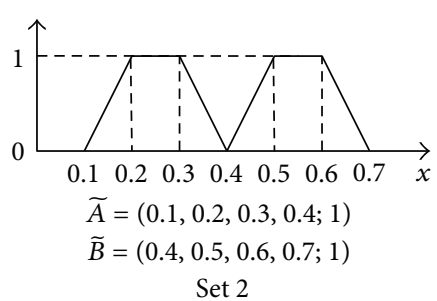

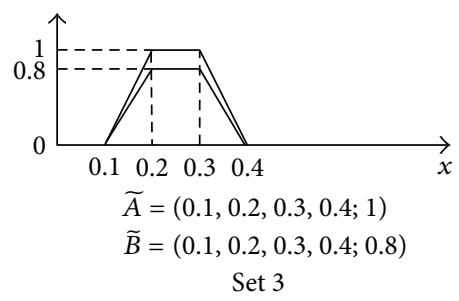

(a)

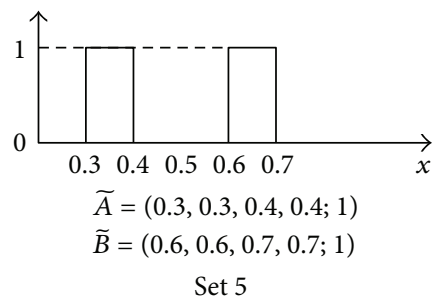

(b)

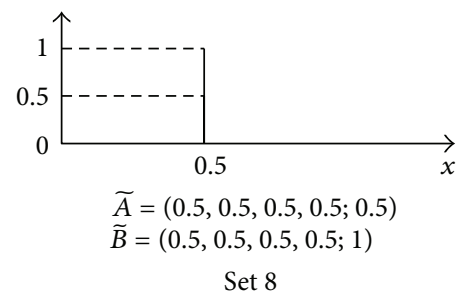

(c)

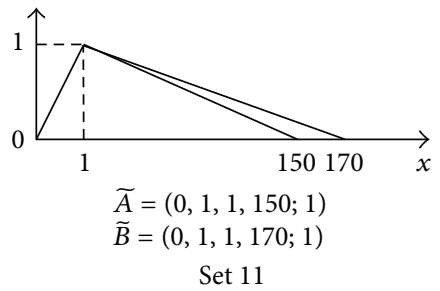

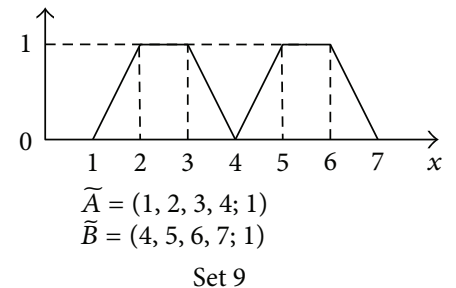

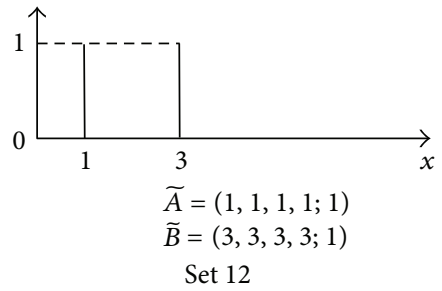

(d)

FIGURE 2: 12 sets of generalized fuzzy numbers.

Some drawbacks of the existing similarity measures are shown in Table 1, which are described as follows.

(1) Set 1 and set 2 of Figure 2 are clearly two different sets of generalized fuzzy numbers. The fuzzy numbers $\widetilde{A}$ and $\widetilde{B}$ in set 2 are more similar than set 1 . However, according to Table 1, Chen's method obtains the same degree of similarity $S(\widetilde{A}, \widetilde{B})$ for set 1 and set 2 , which is an incorrect result.

(2) Set 3 in Figure 2 clearly indicates that the generalized fuzzy numbers $\widetilde{A}$ and $\widetilde{B}$ are not the same, that is, $S(\widetilde{A}, \widetilde{B}) \neq 1$. However, according to Table 1 , Chen's Method and Lee's method obtain the incorrect results, that is, $S(\widetilde{A}, \widetilde{B})=1$.
(3) Set 4 and set 5 of Figure 2 are clearly two different sets of generalized fuzzy numbers. The fuzzy numbers $\widetilde{A}$ and $\widetilde{B}$ in set 5 are more similar than set 4 . However, according to Table 1, Chen's method and Chen and Chen's method obtain the same degree of similarity $S(\widetilde{A}, \widetilde{B})$ for set 4 and set 5 , which are incorrect results. Furthermore, Lee's method obtains an incorrect result, in which the degree of similarity $S(\widetilde{A}, \widetilde{B})$ for set 4 is higher than that of set 5 .

(4) Set 6 in Figure 2 clearly indicates that the generalized fuzzy numbers $\widetilde{A}$ and $\widetilde{B}$ are not the same, that is, $S(\widetilde{A}, \widetilde{B}) \neq 1$. However, according to Table 1 , Chen's method and Lee's method obtain the incorrect results, that is, $S(\widetilde{A}, \widetilde{B})=1$. 
TABLE 1: The comparison of the calculation results of the proposed similarity measure and the existing similarity measures.

\begin{tabular}{|c|c|c|c|c|c|c|}
\hline Sets & Chen's method & Lee's method & Chen and Chen's method & Wei and Chen's method & Wen and Zhou's method & The proposed method \\
\hline Set 1 & 0.7000 & 0.4965 & 0.4200 & 0.6820 & 0.7018 & 0.6567 \\
\hline Set 2 & 0.7000 & 0.5000 & 0.4900 & 0.7000 & 0.7408 & 0.7071 \\
\hline Set 3 & 1 & 1 & 0.8000 & 0.8248 & 0.8324 & 0.8763 \\
\hline Set 4 & 0.7000 & 0.3675 & 0.4900 & 0.6222 & 0.5875 & 0.4743 \\
\hline Set 5 & 0.7000 & 0.2500 & 0.4900 & 0.7000 & 0.7408 & 0.7071 \\
\hline Set 6 & 1 & 1 & 0.7000 & 0.7209 & 0.7291 & 0.8218 \\
\hline Set 7 & 0.9500 & 0.6464 & 0.9048 & 0.6215 & 0.6061 & 0.2810 \\
\hline Set 8 & 1 & \# & 0.5000 & 0.5000 & 0.5000 & 0.5000 \\
\hline Set 9 & -2 & 0.5000 & 4 & -2 & 0.0498 & 0.7071 \\
\hline Set 10 & -4 & 0.9180 & 22.6667 & -3.3481 & 0.0011 & 0.8736 \\
\hline Set 11 & -4 & 0.9412 & 22.6667 & -3.5314 & 0.0011 & 0.9079 \\
\hline Set 12 & -1 & 0 & 1 & -1 & 0.1353 & 0.1353 \\
\hline
\end{tabular}

Note: "\#” means that the similarity measure cannot calculate the degree of similarity between two generalized fuzzy numbers.

"bold" means incorrect results.

TABLE 2: The result of 40 generalized trapezoidal fuzzy numbers $\widetilde{F}_{i j}$.

\begin{tabular}{|c|c|c|c|c|}
\hline \multirow{2}{*}{ Fault feature parameters } & \multirow{2}{*}{\multicolumn{2}{|c|}{$\begin{array}{l}\text { Rotor unbalance } \\
\text { Generalized trapezoidal fuzzy number }\end{array}$}} & \multirow{2}{*}{\multicolumn{2}{|c|}{$\begin{array}{l}\text { Rotor misalignment } \\
\text { Generalized trapezoidal fuzzy number }\end{array}$}} \\
\hline & & & & \\
\hline \multirow{5}{*}{$1 X$} & $\widetilde{X}_{11}$ & $(0.1485,0.1517,0.1770,0.2006 ; 1)$ & $\widetilde{Y}_{11}$ & $(0.1567,0.1600,0.1793,0.1834 ; 1)$ \\
\hline & $\widetilde{X}_{12}$ & $(0.1518,0.1523,0.1669,0.1820 ; 1)$ & $\tilde{Y}_{12}$ & $(0.1644,0.1698,0.1787,0.1809 ; 1)$ \\
\hline & $\widetilde{X}_{13}$ & $(0.1629,0.1635,0.1653,0.1656 ; 1)$ & $\tilde{Y}_{13}$ & $(0.1625,0.1794,0.2069,0.2038 ; 1)$ \\
\hline & $\widetilde{X}_{14}$ & $(0.1606,0.1611,0.1622,0.1630 ; 1)$ & $\tilde{Y}_{14}$ & $(0.1841,0.1878,0.1948,0.1958 ; 1)$ \\
\hline & $\widetilde{X}_{15}$ & $(0.1576,0.1588,0.1607,0.1615 ; 1)$ & $\widetilde{Y}_{15}$ & $(0.1736,0.1773,0.1835,0.1857 ; 1)$ \\
\hline \multirow{5}{*}{$2 X$} & $\widetilde{X}_{21}$ & $(0.1210,0.1426,0.1651,0.1665 ; 1)$ & $\widetilde{Y}_{21}$ & $(0.3071,0.3158,0.3375,0.3466 ; 1)$ \\
\hline & $\widetilde{X}_{22}$ & $(0.1343,0.1457,0.1560,0.1563 ; 1)$ & $\tilde{Y}_{22}$ & $(0.3079,0.3196,0.3361,0.3388 ; 1)$ \\
\hline & $\widetilde{X}_{23}$ & $(0.1420,0.1444,0.1491,0.1534 ; 1)$ & $\tilde{Y}_{23}$ & $(0.3095,0.3269,0.3499,0.3507 ; 1)$ \\
\hline & $\widetilde{X}_{24}$ & $(0.1438,0.1452,0.1486,0.1512 ; 1)$ & $\widetilde{Y}_{24}$ & $(0.3215,0.3287,0.3413,0.3510 ; 1)$ \\
\hline & $\widetilde{X}_{25}$ & $(0.1382,0.1402,0.1453,0.1488 ; 1)$ & $\tilde{Y}_{25}$ & $(0.3132,0.3145,0.3228,0.3291 ; 1)$ \\
\hline \multirow{5}{*}{$3 X$} & $\widetilde{X}_{31}$ & $(0.0899,0.1065,0.1261,0.1296 ; 1)$ & $\widetilde{Y}_{31}$ & $(0.2338,0.2522,0.3022,0.3218 ; 1)$ \\
\hline & $\widetilde{X}_{32}$ & $(0.1044,0.1074,0.1116,0.1131 ; 1)$ & $\widetilde{Y}_{32}$ & $(0.2485,0.2631,0.2820,0.2919 ; 1)$ \\
\hline & $\widetilde{X}_{33}$ & $(0.0990,0.1026,0.1101,0.1150 ; 1)$ & $\widetilde{Y}_{33}$ & $(0.1865,0.1878,0.2556,0.2991 ; 1)$ \\
\hline & $\widetilde{X}_{34}$ & $(0.1059,0.1095,0.1138,0.1158 ; 1)$ & $\tilde{Y}_{34}$ & $(0.2022,0.2078,0.2185,0.2250 ; 1)$ \\
\hline & $\widetilde{X}_{35}$ & $(0.1137,0.1166,0.1199,0.1211 ; 1)$ & $\widetilde{Y}_{35}$ & $(0.2031,0.2120,0.2390,0.2595 ; 1)$ \\
\hline \multirow{5}{*}{ Displacement } & $\widetilde{X}_{41}$ & $(4.1820,4.1933,4.4181,4.6660 ; 1)$ & $\widetilde{Y}_{41}$ & $(4.7450,4.7943,5.1707,5.3920 ; 1)$ \\
\hline & $\widetilde{X}_{42}$ & $(4.3740,4.3917,4.4369,4.4650 ; 1)$ & $\tilde{Y}_{42}$ & $(4.3910,4.4618,4.7070,4.8610 ; 1)$ \\
\hline & $\widetilde{X}_{43}$ & $(4.3190,4.3452,4.3799,4.3890 ; 1)$ & $\widetilde{Y}_{43}$ & $(4.0940,4.0343,4.8373,5.2340 ; 1)$ \\
\hline & $\widetilde{X}_{44}$ & $(4.2800,4.2889,4.3387,4.4000 ; 1)$ & $\widetilde{Y}_{44}$ & $(4.6650,4.7705,5.0505,5.1510 ; 1)$ \\
\hline & $\widetilde{X}_{45}$ & $(4.2870,4.2962,4.3662,4.4620 ; 1)$ & $\widetilde{Y}_{45}$ & $(4.4510,4.4953,4.6309,4.6770 ; 1)$ \\
\hline
\end{tabular}

(5) Set 4 and set 5 of Figure 2 are clearly two different sets of generalized fuzzy numbers. The fuzzy numbers $\widetilde{A}$ and $\widetilde{B}$ in set 5 are more similar than set 4 . However, according to Table 1, Chen and Chen's method obtains an incorrect result, in which the degree of similarity $S(\widetilde{A}, \widetilde{B})$ for set 7 is higher than that of set 6 .

(6) Set 8 in Figure 2 clearly indicates that the degree of similarity between two real values cannot be calculated by Lee's method because the denominator
$\|U\|=\max (U)-\min (U)=0$, which results in the incorrect result $S(\widetilde{A}, \widetilde{B})=\infty$. Furthermore, set 8 in Figure 2 clearly indicates that the generalized fuzzy numbers $\widetilde{A}$ and $\widetilde{B}$ are not the same, that is, $S(\widetilde{A}, \widetilde{B}) \neq 1$. However, Chen's method obtains the incorrect results, that is, $S(\widetilde{A}, \widetilde{B})=1$.

(7) Set 9 in Figure 2 clearly indicates that Chen's method, Chen and Chen's method and Wei and Chen's method cannot deal with nonstandardized fuzzy numbers, 
TABLE 3: The fault template of rotor fault feature.

\begin{tabular}{lcc}
\hline Fault feature & Fault feature parameters & Generalized trapezoidal fuzzy numbers \\
\hline Rotor unbalance $X$ & $1 X$ & $\widetilde{X}_{1}=(0.1563,0.1575,0.1664,0.1745 ; 1)$ \\
& $2 X$ & $\widetilde{X}_{2}=(0.1359,0.1436,0.1528,0.1552 ; 1)$ \\
& $3 X$ & $\widetilde{X}_{3}=(0.1026,0.1085,0.1163,0.1189 ; 1)$ \\
Rotor misalignment $Y$ & Displacement & $\widetilde{X}_{4}=(4.2884,4.3031,4.3880,4.4764 ; 1)$ \\
\hline
\end{tabular}

TABLE 4: The diagnosing rotor fault feature.

\begin{tabular}{lc}
\hline Fault feature parameters & Generalized trapezoidal fuzzy numbers \\
\hline $1 X$ & $\widetilde{A}_{1}=(0.1515,0.1550,0.1743,0.1784 ; 1)$ \\
$2 X$ & $\widetilde{A}_{2}=(0.3221,0.3308,0.3525,0.3611 ; 1)$ \\
$3 X$ & $\widetilde{A}_{3}=(0.1338,0.1522,0.2021,0.2214 ; 1)$ \\
Displacement & $\widetilde{A}_{4}=(4.2450,4.2943,4.6707,4.8910 ; 1)$ \\
\hline
\end{tabular}

which all give the incorrect result, that is, $S(\widetilde{A}, \widetilde{B}) \notin$ $[0,1]$. Moreover, set 9 has the same degree of similarity $S(\widetilde{A}, \widetilde{B})$ as set 2 , because the geometric distance, the center of gravity point, the perimeter, and the area of the generalized trapezoidal fuzzy numbers of the generalized trapezoidal fuzzy numbers in set 9 should be equal to those in set 2 after normalization process. However, Wen and Zhou's Method obtains an incorrect result, in which the degree of similarity $S(\widetilde{A}, \widetilde{B})$ for set 9 is particularly lower than that of set 2 .

(8) Set 10 and set 11 of Figure 2 clearly indicates that Chen's method, Chen and Chen's Method and Wei and Chen's method cannot deal with nonstandardized fuzzy numbers, which all give the incorrect result, that is, $S(\widetilde{A}, \widetilde{B}) \notin[0,1]$. Moreover, set 10 and set 11 of Figure 2 are clearly two different sets of nonstandardized generalized fuzzy numbers. Nonstandardized generalized fuzzy numbers $\widetilde{A}$ and $\widetilde{B}$ in set 11 are more similar than set 10 . However, according to Table 1, Wen and Zhou's method obtains the same degrees of similarity $S(\widetilde{A}, \widetilde{B})$ for set 10 and set 11 . Finally, set 10 and set 11 in Figure 2 clearly indicates that nonstandardized fuzzy numbers $\widetilde{A}$ and $\widetilde{B}$ are approximately similar, that is, $S(\widetilde{A}, \widetilde{B})>0.5$. But Wen and Zhou's method obtains the incorrect results, that is, $S(\widetilde{A}, \widetilde{B}) \ll 0.5$.

(9) Set 12 in Figure 2 clearly indicates that Chen's method, Lee's method, and Wei and Chen's method cannot deal with nonstandardized fuzzy numbers, which all give the incorrect result, that is, $S(\widetilde{A}, \widetilde{B}) \notin[0,1]$. Moreover, set 12 in Figure 2 clearly indicates that nonstandardized generalized fuzzy numbers $\widetilde{A}$ and $\widetilde{B}$ are not same, that is, $S(\widetilde{A}, \widetilde{B}) \neq 1$. However, Chen and
Chen's method obtains the incorrect results, that is, $S(\widetilde{A}, \widetilde{B})=1$.

From Table 1 and Figure 2, it clearly indicates that the proposed similarity measure can overcome the drawbacks of the existing similarity measures.

\section{Rotor Fault Diagnosis Based on the Proposed Similarity Measure}

In this section, the proposed similarity measure is used to deal with the rotor fault diagnosis problem. The major cause of machine vibration is rotor unbalance and misalignment, which leads to additional dynamic load and to accelerate machine deterioration [23]. The proposed similarity measure is a very helpful method in diagnosing the rotor unbalance and misalignment to avoid any failures or damages that may arise.

The proposed similarity measure will be verified by fault diagnosis experiments on multifunctional flexible rotor experiment bench. The vibration acceleration and vibration displacement sensors are, respectively, installed on supporting rotor bases to collect vibration signals by data collector. Experimental procedures involve following main steps.

6.1. Determine the Rotor Fault Diagnosis Set. Rotor unbalance and misalignment are two major rotor fault features. We determine the rotor fault diagnosis set as $F=\{X, Y\}$, where $X=$ rotor unbalance and $Y=$ rotor misalignment.

6.2. Structure the Fault Template of Rotor Fault Feature. Rotor speed is $1500 \mathrm{rpm}$, and we choose $1 X, 2 X$, and $3 X$ amplitude frequency response (fundamental frequency is $25 \mathrm{~Hz}$ ) and average amplitude of vibration displacement as four types of rotor fault feature parameters. In order to structure the fault template of rotor fault feature, we adopt experimental statistics method to simulate rotor fault operation mode and 
TABLE 5: Rotor fault diagnosis result of Wen and Zhou's method.

\begin{tabular}{llcc}
\hline \multirow{2}{*}{ Fault feature parameters } & \multicolumn{2}{c}{ The degree of similarity } & Rotor fault diagnosis result \\
\hline $1 X$ & $S\left(\widetilde{A}_{1}, \widetilde{X}_{1}\right)=0.9853$ & Rotor misalignment $Y$ & Rotor unbalance \\
$2 X$ & $S\left(\widetilde{A}_{2}, \widetilde{X}_{2}\right)=0.8038$ & $S\left(\widetilde{A}_{1}, \widetilde{Y}_{1}\right)=0.9769$ & Rotor misalignment \\
$3 X$ & $S\left(\widetilde{A}_{3}, \widetilde{X}_{3}\right)=0.8638$ & $S\left(\widetilde{A}_{2}, \widetilde{Y}_{2}\right)=0.9776$ & Rotor misalignment \\
Displacement & $S\left(\widetilde{A}_{4}, \widetilde{X}_{4}\right)=0.5756$ & $S\left(\widetilde{A}_{3}, \widetilde{Y}_{3}\right)=0.9105$ & Rotor misalignment \\
\hline
\end{tabular}

TABLE 6: Rotor fault diagnosis result of the proposed method.

\begin{tabular}{lccc}
\hline \multirow{2}{*}{ Fault feature parameters } & \multicolumn{2}{c}{ The degree of similarity } & Rotor fault diagnosis result \\
& Rotor unbalance $X$ & $S\left(\widetilde{A}_{1}, \widetilde{Y}_{1}\right)=0.6512$ & Rotor misalignment \\
$1 X$ & $S\left(\widetilde{A}_{1}, \widetilde{X}_{1}\right)=0.6404$ & $S\left(\widetilde{A}_{2}, \widetilde{Y}_{2}\right)=0.7145$ & Rotor misalignment \\
$2 X$ & $S\left(\widetilde{A}_{2}, \widetilde{X}_{2}\right)=0.6961$ & $S\left(\widetilde{A}_{3}, \widetilde{Y}_{3}\right)=0.6350$ & Rotor misalignment \\
$3 X$ & $S\left(\widetilde{A}_{3}, \widetilde{X}_{3}\right)=0.4282$ & $S\left(\widetilde{A}_{4}, \widetilde{Y}_{4}\right)=0.7797$ & Rotor misalignment \\
Displacement & $S\left(\widetilde{A}_{4}, \widetilde{X}_{4}\right)=0.4617$ &
\end{tabular}

collect five sets of data for each type of rotor fault feature parameter. Every set of data consists of 40 observations. Let $F_{i j}=\left[f_{j, 1}, f_{j, 2}, \ldots, f_{j, 40}\right]$ indicate the 40 observations of the $j$ set of data within 16s, where $F=\{X, Y\}$ represent two rotor fault features, respectively, $i=1,2,3,4$ represent four types of rotor fault feature parameters, respectively, and $j=1,2,3,4,5$ represent the set number of the collected data. The arithmetic average $M_{j}$ and mean deviation $\sigma_{j}$ of the $j$ set of data can be got as follows:

$$
\begin{gathered}
M_{j}=\frac{\left(f_{j, 1}+f_{j, 2}+\cdots+f_{j, 40}\right)}{40}, \\
\sigma_{j}=\sqrt{\frac{\left(\left(f_{j, 1}-M_{j}\right)^{2}+\left(f_{j, 2}-M_{j}\right)^{2}+\cdots+\left(f_{j, 40}-M_{j}\right)^{2}\right)}{40}} .
\end{gathered}
$$

Then, the generalized trapezoidal fuzzy number $\widetilde{F}_{i j}=$ $\left(f_{1}, f_{2}, f_{3}, f_{4}, w_{\widetilde{F}}\right)$ can be constructed based on these collected data as follows:

$$
\begin{gathered}
f_{1}=\min \left(f_{j, k}\right), \\
f_{2}=M_{j}-\sigma_{j}, \\
f_{3}=M_{j}+\sigma_{j}, \\
f_{4}=\max \left(f_{j, k}\right), \\
w_{\widetilde{F}}=1 .
\end{gathered}
$$

We can obtain 40 generalized trapezoidal fuzzy numbers $\widetilde{F}_{i j}$, where $\widetilde{F}_{i j}=\left\{\widetilde{X}_{i j}, \widetilde{Y}_{i j}\right\}, i=1,2,3,4$ and $j=1,2,3,4,5$. The result of 40 generalized trapezoidal fuzzy numbers $\widetilde{F}_{i j}$ is shown in Table 2.
The generalized trapezoidal fuzzy number for each fault feature parameter can be got as follows:

$$
\widetilde{F}_{i}=\frac{\sum_{j=1}^{5} \widetilde{F}_{i j}}{5} .
$$

The result of generalized trapezoidal fuzzy numbers for the fault template is shown in Table 3.

6.3. Structure the Diagnosing Rotor Fault Feature. We let multifunctional flexible rotor experiment bench bed run in rotor misalignment mode $Y$ and collect data for each parameter of rotor fault feature. The generalized trapezoidal fuzzy numbers $\widetilde{A}_{i}$ of diagnosing rotor fault feature can be obtained through the collected data. The result is shown in Table 4.

6.4. Calculate the Degree of Similarity between Fuzzy Numbers $\widetilde{A}_{i}$ and Each Fault Feature Parameter. In the following, Wen and Zhou's and the proposed methods are used to deal with the rotor fault diagnosis problem, respectively. Based on (11) and (14), the degree of similarity between generalized trapezoidal fuzzy numbers $\widetilde{A}_{i}$ and each fault feature parameter shown in Table 3 can be calculated. The calculating rotor fault diagnosis results of Wen and Zhou's and the proposed methods are shown in Tables 5 and 6, respectively.

From Table 5 , we obtain $S\left(\widetilde{A}_{1}, \widetilde{X}_{1}\right)>S\left(\widetilde{A}_{1}, \widetilde{Y}_{1}\right), S\left(\widetilde{A}_{2}\right.$, $\left.\widetilde{X}_{2}\right)<S\left(\widetilde{A}_{2}, \widetilde{Y}_{2}\right), S\left(\widetilde{A}_{3}, \widetilde{X}_{3}\right)<S\left(\widetilde{A}_{3}, \widetilde{Y}_{3}\right)$, and $S\left(\widetilde{A}_{4}, \widetilde{X}_{4}\right)<$ $S\left(\widetilde{A}_{4}, \widetilde{Y}_{4}\right)$. It means that the rotor fault diagnosis result for $1 X$ fault feature parameter is rotor unbalance and the result for the other three types of rotor fault feature parameters are rotor misalignment. Thus, it is unable to determine fault feature from the rotor fault diagnosis result of Wen and Zhou's method. Comparing with the result of Wen and Zhou's method, the rotor fault diagnosis results of the proposed method for four types of rotor fault feature parameters are all rotor misalignment. The rotor fault diagnosis result of 
the proposed method coincides with the fault feature of multifunctional flexible rotor experiment bench. Therefore, experimental results demonstrate that the proposed similarity measure is more effective than Wen and Zhou's methods in terms of rotor fault diagnosis.

\section{Conclusions}

This paper presents a new similarity measure used for fault diagnosis technology, which can deal with both standardized and nonstandardized generalized trapezoidal fuzzy numbers. It combines concepts of the geometric distance, the center of gravity point, the perimeter, and the area of generalized trapezoidal fuzzy numbers for calculating the degree of similarity $S(\widetilde{A}, \widetilde{B})$ between $\widetilde{A}$ and $\widetilde{B}$. Some properties of the proposed similarity measure have been proved, and 12 sets of generalized fuzzy numbers have been used to compare the calculation results of the proposed similarity measures with the existing similarity measures. From Table 1 and Figure 2, it clearly indicates that the proposed similarity method can overcome the drawbacks of existing similarity measures. Finally, a rotor fault diagnosis example has been given to show the proposed similarity measure.

\section{Acknowledgment}

The authors would like to thank the anonymous reviewers for critical comments and suggestions leading to an improved presentation.

\section{References}

[1] A. T. Ghainani, A. A. M. Zin, and N. M. Ismail, "Fuzzy timing petri net for fault diagnosis in power system," Mathematical Problems in Engineering, vol. 2012, Article ID 717195, 12 pages, 2012.

[2] C. Roberts, H. P. B. Dassanayake, N. Lehrasab, and C. J. Goodman, "Distributed quantitative and qualitative fault diagnosis: railway junction case study," Control Engineering Practice, vol. 10, no. 4, pp. 419-429, 2002.

[3] R. Ubar, S. Kostin, and J. Raik, "Embedded fault diagnosis in digital systems with BIST," Microprocessors and Microsystems, vol. 32, no. 5-6, pp. 279-287, 2008.

[4] S.-J. Chen and S.-M. Chen, "Fuzzy risk analysis based on similarity measures of generalized fuzzy numbers," IEEE Transactions on Fuzzy Systems, vol. 11, no. 1, pp. 45-56, 2003.

[5] R. Eslamloueyan, "Designing a hierarchical neural network based on fuzzy clustering for fault diagnosis of the TennesseeEastman process," Applied Soft Computing, vol.11, pp. 1407-1415, 2011.

[6] H. Luo, S. L. Yang, X. J. Hu, and X. X. Hu, "Agent oriented intelligent fault diagnosis system using evidence theory," Expert Systems with Applications, vol. 39, pp. 2524-2531, 2012.

[7] Z. Du, X. Jin, and Y. Yang, "Fault diagnosis for temperature, flow rate and pressure sensors in VAV systems using wavelet neural network," Applied Energy, vol. 86, no. 9, pp. 1624-1631, 2009.

[8] V. Muralidharan and V. Sugumaran, "Feature extraction using wavelets and classification through decision tree algorithm for fault diagnosis of mono-block centrifugal pump," Measurement, vol. 46, pp. 353-359, 2013.
[9] J. Zhang, "Improved on-line process fault diagnosis through information fusion in multiple neural networks," Computers and Chemical Engineering, vol. 30, pp. 558-571, 2006.

[10] M. Grbovic, W. C. Li, P. Xu, A. K. Usadi, L. M. Song, and S. Vucetic, "Decentralized fault detection and diagnosis via sparse PCA based decomposition and Maximum Entropy decision fusion," Journal of Process Control, vol. 22, pp. 738-750, 2012.

[11] M. Barakat, F. Druaux, D. Lefebvre, M. Khalil, and O. Mustapha, "Self adaptive growing neural network classifier for faults detection and diagnosis," Neurocomputing, vol. 74, pp. 38653876, 2011.

[12] M. Weerasinghe, J. Barry Gomm, and D. Williams, "Neural networks for fault diagnosis of a nuclear fuel processing plant at different operating points," Control Engineering Practice, vol. 6, no. 2, pp. 281-289, 1998.

[13] X. B. Xu, H. S. Feng, Z. Wang, and C. L. Wen, "An information fusion method of fault diagnosis based on interval basic probability assignment," Chinese Journal of Electronics, vol. 20, no. 2, pp. 255-260, 2011.

[14] S.-J. Chen, "New methods for subjective mental workload assessment and fuzzy risk analysis," Cybernetics and Systems, vol. 27, no. 5, pp. 449-472, 1996.

[15] H. S. Lee, "Optimal aggregation method for fuzzy opinions of group decision," in Proceedings of the IEEE International Conference on Systems, Man, and Cybernetics (SMC '99), vol. 3, pp. 314-319, October 1999.

[16] S.-J. Chen and S.-M. Chen, "A new method to measure the similarity between fuzzy numbers," in Proceedings of the 10th IEEE International Conference on Fuzzy Systems, pp. 208-214, Melbourne, Australia, 2011.

[17] J. H. Chen and S.-M. Chen, "A new method for ranking generalized fuzzy numbers for handling fuzzy risk analysis problems," in Proceedings of the 9th Joint Conference on Information Sciences (JCIS '06), pp. 1196-1199, Kaohsiung, Taiwan, October 2006.

[18] S.-J. Chen and S.-M. Chen, "Fuzzy risk analysis based on the ranking of generalized trapezoidal fuzzy numbers," Applied Intelligence, vol. 26, no. 1, pp. 1-11, 2007.

[19] S.-H. Wei and S.-M. Chen, "A new similarity measure between generalized fuzzy numbers," in Proceedings of the 3rd International Conference on Soft Computing and Intelligent System and 7th International Symposium on Advanced Intelligent System, pp. 315-320, Tokyo, Japan, 2006.

[20] S.-H. Wei and S.-M. Chen, "A new approach for fuzzy risk analysis based on similarity measures of generalized fuzzy numbers," Expert Systems with Applications, vol. 36, no. 1, pp. 589-598, 2009.

[21] C. L. Wen, Z. Zhou, and X. B. Xu, "A new similarity measure between generalized trapezoidal fuzzy numbers and its application to fault diagnosis," Acta Electronica Sinica, vol. 39, no. 3, pp. $1-6,2011$

[22] S.-H Chen, "Ranking generalized fuzzy number with graded mean integration," in Proceedings of the 8th international Fuzzy Systems Association World Congress, vol. 2, pp. 899-902, Taipei, Taiwan, 1999.

[23] L. Arebi, F. Gu, and A. Ball, "Rotor Misalignment detection using a wireless sensor and a shaft encoder," in Proceedings of the Computing and Engineering Annual Researchers' Conference (CEARC '10), pp. 6-13, University of Huddersfield, Huddersfield, UK, 2010. 


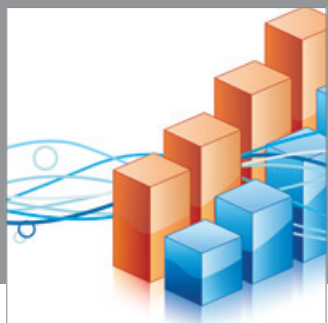

Advances in

Operations Research

mansans

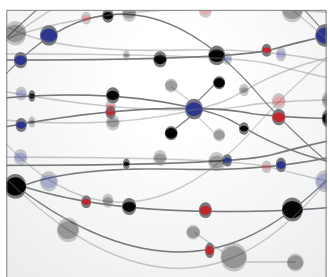

The Scientific World Journal
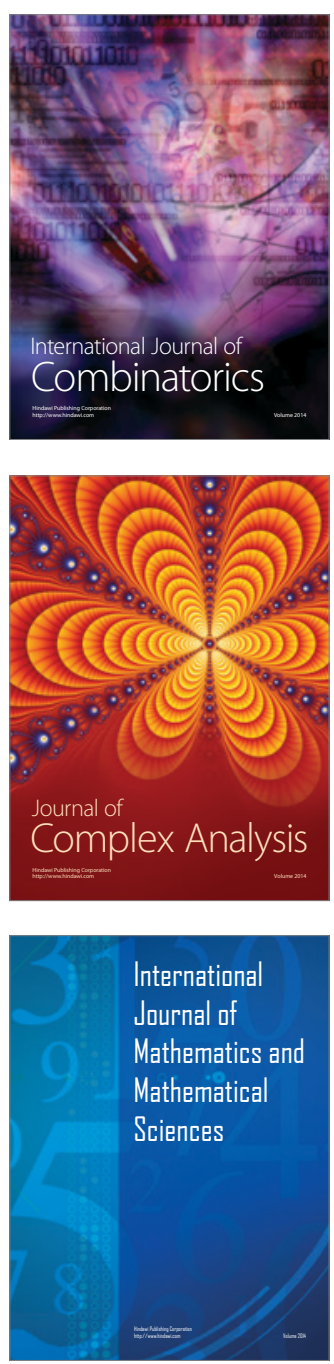
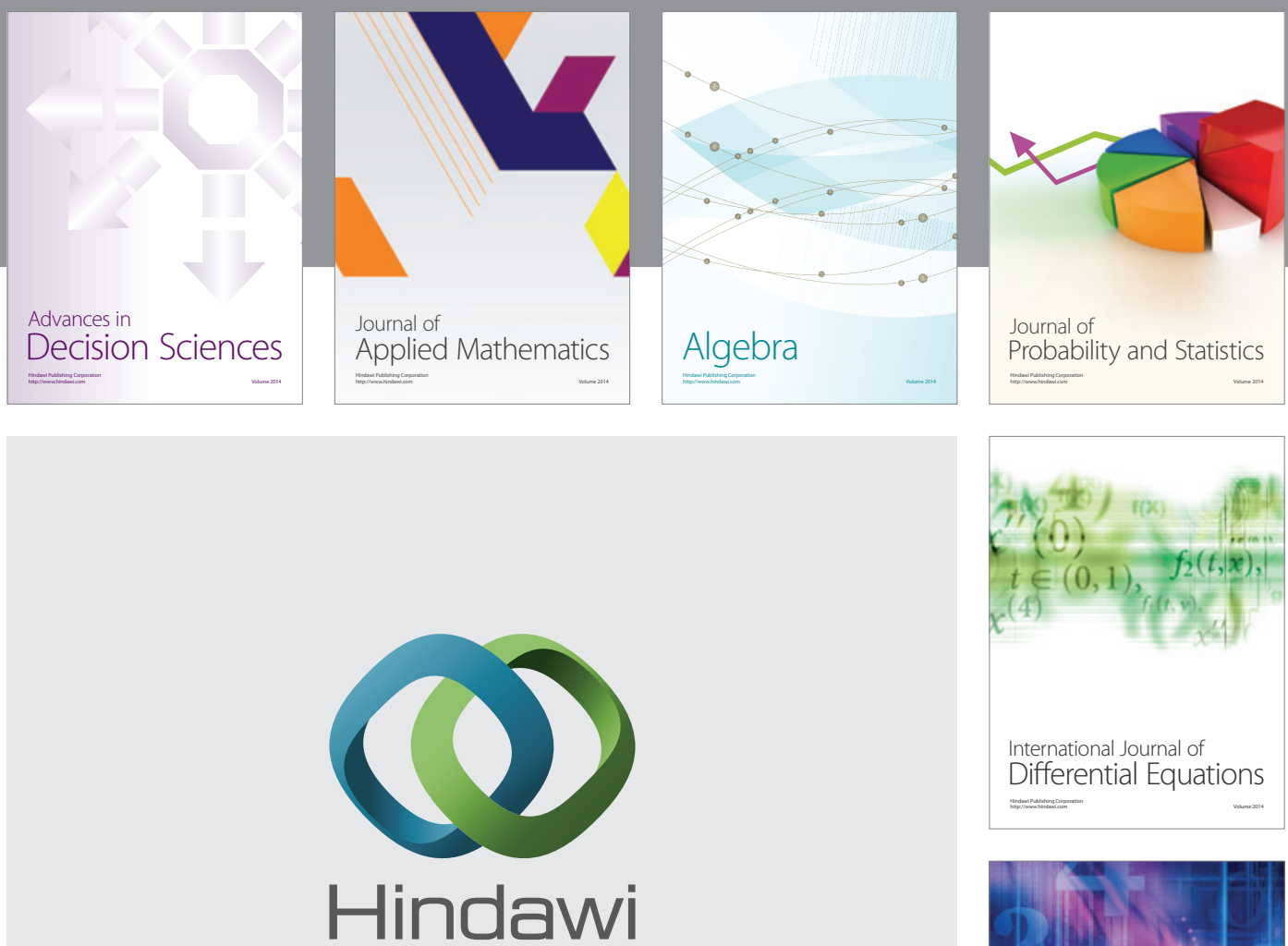

Submit your manuscripts at http://www.hindawi.com
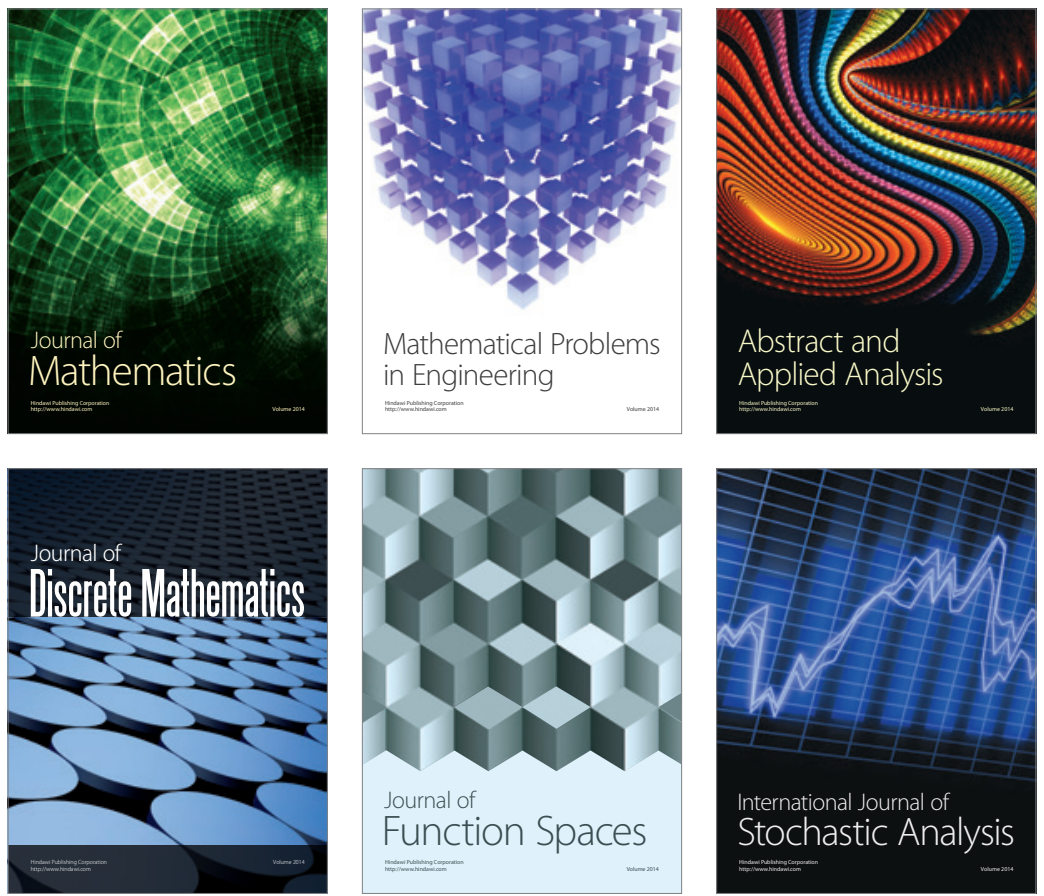

Journal of

Function Spaces

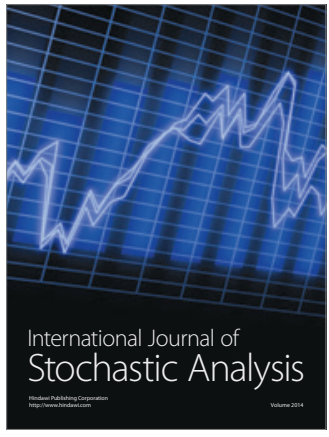

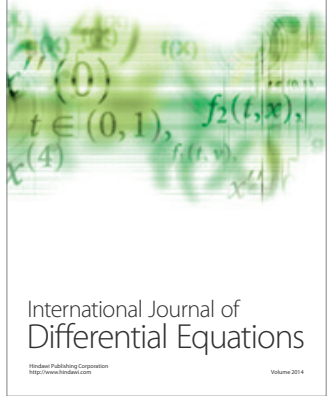
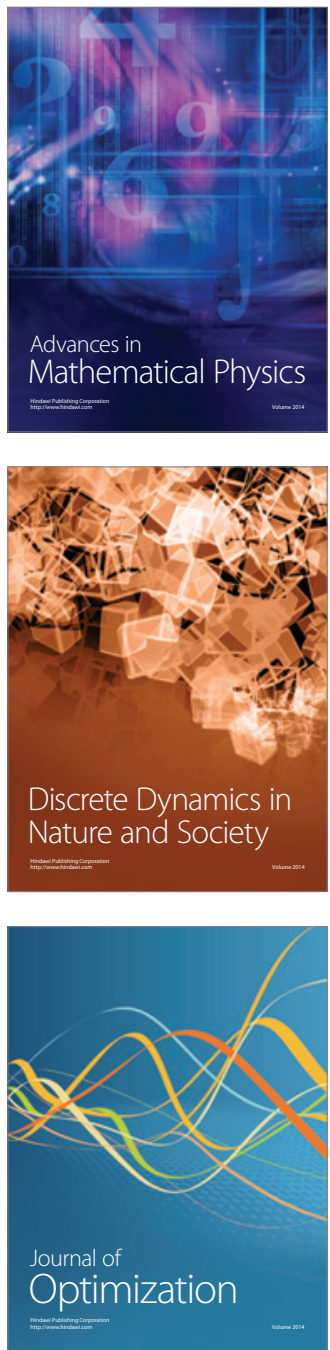